\title{
MINIMAL SIZE REDUCTION IN PLANARIANS THROUGH SUCCESSIVE REGENERATIONS
}

\section{S. J. HOLMES}

It is a familiar fact that very small pieces of fresh water plan arians will regenerate and give rise to minute individuals closely resembling the original form. I have endeavored to ascertain how far reduction of size in Planaria maculata may be carried without causing a failure to give rise to a normal individual. With very small pieces of an adult planarian there is a large proportion of cut surface which produces an injurious effect and there are also various mechanical impediments to regeneration; these facts, combined with the specialized condition of much of the tissue, conspire to restrict the regenerative capacity of the parts. In order to eliminate somewhat these factors the device was resorted to of subjecting the animals to a number of successive divisions. A planarian was cut into fifteen or twenty pieces; when these had regenerated into small planarians they were again cut into several pieces. These regenerated into still smaller individuals which in turn were divided, the process being con. tinued until forms were reached which were so small that complete regenerations were no longer obtained. In this way, wheli the minimal size limit was approached, regeneration became very slow and many of the pieces lived for months without restoration of the missing parts. As a general rule it may be said that the smaller the piece the more slowly the restorative processes take place. In this way the proportion of cut surface was reduced, the tissue kept in a more plastic condition, and the whole process of regeneration made easier.

Eugene Schultz has studied the reduction in size of planarians from starvation. He found that planarians could be reduced in this way to one-tenth or one-twelfth of their original size. A study of the size of cells of various kinds showed that there was 
little reduction in size either of the cells or their nuclei as a result of starvation; the diminution in the size of the body was produced mainly by their reduction in number. Various organs suffered unequally in this process. Organs of copulation, sex ducts and vitellaria were among the first to disappear, the eyes degenerated, and there was a marked reduction in the number of parenchyma cells. The muscle cells suffered little decrease and the number of muscle bands remained unaltered; there was little reduction in the nervous system. The male sex cells were among the least altered. Cells of the intestinal epithelium and the outer ectoderm, while reduced in number, were not reduced disproportionally to the body as a whole.

The study of small regenerated planarians was undertaken in order to ascertain how far the various organ systems would suffer on account of reduction in size and how far the results might be parallel with the effects of starvation. Through successive regenerations it is possible to carry the reduction very much farther than can be done by the withdrawal of food. While starvation may reduce the animal to one-tenth or one-twelfth its original size, by the method of successive regenerations it may be reduced to 1000 or $\frac{1}{1000}$ its original size. Many of these very minute animals had practically the same form as the adult. Several specimens were sectioned and careful measurements were made of several kinds of cells and compared with measurements of corresponding cells of individuals of ordinary size. Ectoderm cells, parenchyma cells, cells of the intestinal epithelium were of the same size as in the larger worms. The muscle cells, while less in length, were nearly as thick as in the larger worms. The nuclei were also not reduced in size and therefore bore the usual relation to the size of the cells. The gonads, sex ducts, copulatory apparatus and vitellaria could not be found. The muscular system is well developed, the outer layers being present and only a little thinner than in normal individuals. The number of dorsoventral strands in a cross-section is not more than about one-fourth that of larger specimens and they contain fewer fibers. The alimentary canal has but a very few short branches. The cells are not shrunken as occurs in starved individuals and 
cross sections of the diverticula appear much as in the larger worms except for the reduction in the number of cells. The size of the brain and the diameter of the nerve cords bear about the same ratio to the rest of the body as in large individuals. In a few cases there was but one cye instead of two and this was not a median one as sometimes occurs in small individuals but was in the position of one of the lateral eyes. The cye is of normal size, in relation to other parts and it has essentially the usual structure, but there is a great reduction of the number of the retinal cells.

The pharynx, which bears about the same relative proportion to the body as in larger planarians, is composed of the same epithelial and muscular layers. The relative proportion of the parenchyma and digestive organs is little altered in the smaller individuals. The relative thickness of the outer epithelium is however much greater, since it is composed of but one layer of cells which have the same size in the large and the small planarians. Pigment cells occur sparsely scattered over the dorsal surface and appear of enormous size in relation to the rest of the body. On the whole, the small individuals are strikingly like the larger ones in general form and the relative proportions of the systems of organs.

Observations were made on the movements and reactions of these minute forms. Methods of locomotion, exploring movements of the head, reactions to light and contact, responses to mechanical stimuli, righting movements, and various other activities, even down to the most delicate details, were carried out in practically the same way as in individuals of normal size. These facts indicate how effectively the functional unity of the organism is maintained notwithstanding the enormous reduction in the number of its cells.

One factor which probably determines the mininal size which may be attained is the fact that the size of the cells cannot be reduced and there must be a certain number of kinds of cells to preserve the physiological unity of the organism. There must be nerve cells, muscle cells, parenchyma cells, epithelium, etc., if the planarian is to be a planarian. The work of the organism, like 
that of a factory, can be performed on a large or a small scale, but as there are many functions to be discharged, and as one cell cannot do the work of another, a point naturally has to be reached somewhere when a further reduction of the number of cells brings operations to a standstill. Matters might work out, however, in a different way by effecting a general simplification of structure, such as occurs in the reduction of Hydra. This simplification of structure, which has been compared to a reversal of embryonic development, does not proceed in the planarian very far. The loss of sex ducts and associated organs is of doubtful significance, since these parts often atrophy at certain periods in adult individuals. In attempting to carry reduction below a certain size the cut ends of the pieces heal over and there is little further change; the organism does not transform itself into a simple embryonic stage, and we cannot with safety speak of the reversal of developmental processes (if it be really such) beyond perhaps a few retrogressive steps. 